\title{
Experimental hypercholesterolemia differentially affects adventitial vasa vasorum and vessel structure of the left internal thoracic and coronary arteries
}

\author{
Offer Galili, MD \\ Katherine J. Sattler, MD \\ Joerg Herrmann, MDa \\ Julie Woodrum, MS ${ }^{\mathrm{a}}$ \\ Monica Olson, BS ${ }^{\mathrm{a}}$ \\ Lilach 0. Lerman, MD, $\mathrm{PhD}^{\mathrm{b}}$ \\ Amir Lerman, MD ${ }^{\mathrm{a}}$
}

From the Divisions of Cardiovascular Dis-
eases $^{\mathrm{a}}$ and Nephrology and Hypertension,
Mayo College of Medicine, Rochester,
Minn.
This work was supported by the National
Institutes of Health (R01 HL 63911, K-24
HL 69840-02, and R01 HL63282), the Mi-
ami Heart Research Institute, and the Mayo
Foundation. Dr Amir Lerman is an Estab-
lished Investigator of the American Heart
Association.

Received for publication April 22, 2004; revisions received July 13, 2004; accepted for publication Aug 15, 2004.

Address for reprints: Amir Lerman, MD, Division of Cardiovascular Diseases, Mayo Clinic Rochester, 200 First St SW, Rochester, MN, 55905 (E-mail: lerman.amir@ mayo.edu).

J Thorac Cardiovasc Surg 2005;129:767-72 0022-5223/\$30.00

Copyright (C) 2005 by The American Association for Thoracic Surgery

doi:10.1016/j.jtcvs.2004.08.014
Objective: Atherosclerosis is a chronic and diffuse disease that affects all vascular beds. However, some vascular beds are more prone to atherosclerosis than others. Recent evidence suggests a role for the vasa vasorum in the atherosclerotic process. We hypothesized that there is a difference in adventitial vasa vasorum structure between the left internal thoracic artery and the coronary artery. Hence the current study was designed to characterize and compare the structure of the adventitial vasa vasorum in the left internal thoracic and coronary arteries.

Methods: Samples of vessels were obtained from female crossbred domestic pigs maintained on a normal $(n=6)$ or high-cholesterol $(n=6)$ diet for 12 weeks. The samples were scanned with micro-computed tomography, and the tomographic images were reconstructed and analyzed to obtain lumen area, vessel wall area, vasa vasorum count, vasa vasorum density, mean diameter of first- and second-order vasa vasorum, and second-order/first-order vasa vasorum ratio.

Results: Vasa vasorum density was significantly higher in the coronary arteries versus that seen in the left internal thoracic arteries in the normal group, as well as in the high-cholesterol group. The higher vasa vasorum density in the highcholesterol group versus that in the normal group was significant for both vessels, being more pronounced in the left internal thoracic artery. Lumen area and secondorder/first-order vasa vasorum ratio were higher in the high-cholesterol group than in the normal group only in the left internal thoracic artery.

Conclusion: This study demonstrated that low vasa vasorum spatial density and higher lumen area observed in the left internal thoracic artery compared with that seen in the native coronary artery can be the structural background for the low incidence of atherosclerosis in this vessel.

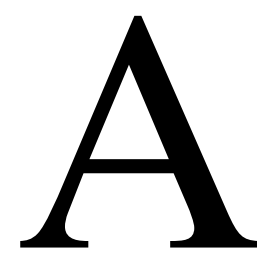

therosclerosis is a chronic and diffuse disease that affects all vascular beds. However, it is apparent that some vascular beds are more prone to atherosclerosis than others. The coronary vasculature is the most affected by the atherosclerotic process, as reflected in the data recently published by the American Heart Association $^{1}$ and supported by autopsy-based studies. ${ }^{2,3}$ In contrast, the left internal thoracic artery (LITA) is well known for its low incidence of atherosclerosis, ${ }^{4}$ a biologic characteristic that makes it an excellent conduit for 


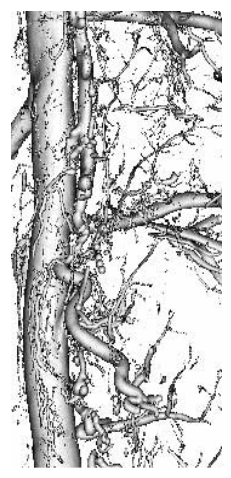

N-Coronary

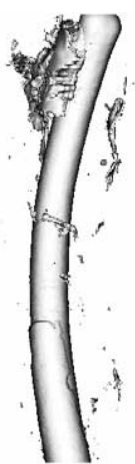

N-LITA

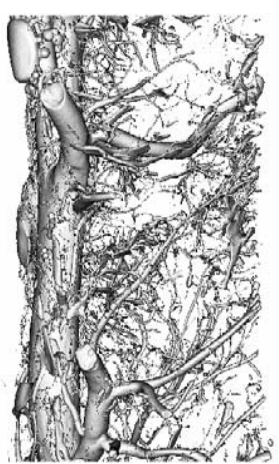

HC-Coronary

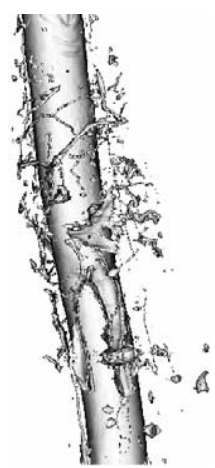

HC-LITA

Figure 1. Representative micro-CT images of the coronary artery and the left internal thoracic artery (LITA) of pigs maintained on a normal diet (N) and a high-cholesterol diet (HC) for 12 weeks.

TABLE 1. Micro-CT analysis data: Morphologic characteristic of the LITA and coronary vessels from pigs maintained on normal or high-cholesterol diets for 12 weeks (mean $\pm \mathrm{SE}$ )

\begin{tabular}{|c|c|c|c|c|c|c|}
\hline & \multicolumn{3}{|c|}{ Normal diet } & \multicolumn{3}{|c|}{ High-cholesterol diet } \\
\hline & Coronary artery & LITA & $P$ value & Coronary artery & LITA & $P$ value \\
\hline \multicolumn{7}{|l|}{ Host vessel dimensions } \\
\hline Lumen area ( $\mathrm{mm}^{2}$ mean/section) & $3.11 \pm 0.19$ & $3.6 \pm 0.29$ & .364 & $3.19 \pm 0.11$ & $5.13 \pm 0.14^{*}$ & $<.001$ \\
\hline $\begin{array}{l}\text { Vessel wall area (mm² mean/ } \\
\text { section) }\end{array}$ & $2.1 \pm 0.08$ & $7.18 \pm 0.24$ & $<.001$ & $2.97 \pm 0.2 \dagger$ & $9.36 \pm 0.48^{*}$ & $<.001$ \\
\hline \multicolumn{7}{|l|}{ Vasa vasorum dimensions } \\
\hline $\begin{array}{l}\text { Vasa vasorum count (n mean/ } \\
\text { section) }\end{array}$ & $6.45 \pm 0.63$ & $1.55 \pm 0.24$ & $<.001$ & $13.6 \pm 0.7 \dagger$ & $8.56 \pm 0.42^{*}$ & $<.001$ \\
\hline $\begin{array}{l}\text { Vasa vasorum density }\left(\mathrm{n} / \mathrm{mm}^{2}\right. \\
\text { mean/section) }\end{array}$ & $2.91 \pm 0.26$ & $0.22 \pm 0.04$ & $<.001$ & $5.09 \pm 0.37 \dagger$ & $1.01 \pm 0.06^{*}$ & $<.001$ \\
\hline $\begin{array}{l}\text { Second-order/first-order vasa } \\
\text { vasorum ratio (mean/section) }\end{array}$ & $4.22 \pm 0.46$ & $0.84 \pm 0.2$ & $<.001$ & $4.25 \pm 0.31$ & $5.77 \pm 0.52^{*}$ & .022 \\
\hline $\begin{array}{l}\text { First-order vasa vasorum } \\
\text { diameter }(\mu \mathrm{m})\end{array}$ & $84.17 \pm 2.4$ & $79.28 \pm 7.34$ & .02 & $97.28 \pm 5.41$ & $107.2 \pm 4.27^{*}$ & .057 \\
\hline $\begin{array}{l}\text { Second-order vasa vasorum } \\
\text { diameter }(\mu \mathrm{m})\end{array}$ & $58.1 \pm 2.2$ & $57.4 \pm 5.7$ & .09 & $55.67 \pm 1.23$ & $67.6 \pm 1.9^{*}$ & $<.001$ \\
\hline
\end{tabular}

$* P<.001$ versus LITA, normal diet.

$\dagger P<.001$ versus coronary artery, normal diet.

coronary artery bypass grafting. ${ }^{5}$ However, the inherent features responsible for the differential vascular propensity for atherosclerosis remain unclear.

Traditionally, the outer wall of the vessel, which includes the adventitial layer and the vasa vasorum (VV), has been ascribed a passive role in the pathologic process of atherosclerosis. However, recent evidence suggests that these structures might play a significant role in maintaining vessel integrity and might contribute to the initiation and progression of atherosclerosis. ${ }^{6}$ Indeed, experimental studies demonstrated that manipulation of the adventitia and, more specifically, of the VV could lead to atherosclerotic changes of the intima. ${ }^{7}$ Vice versa, atherosclerotic lesion formation is associated with remarkable neovascularization of the
VV. ${ }^{8}$ Using unique micro-computed tomography (microCT) technology, ${ }^{9}$ we were recently able to outline the 3dimensional anatomy of the VV in normal and hypercholesterolemic porcine coronary arteries, showing an enhanced VV neovascularization in hypercholesterolemia. ${ }^{10}$ The increase in the spatial density of the adventitial VV precedes endothelial dysfunction, suggesting a significant role for VV proliferation in the early atherosclerotic remodeling process before functional alterations and plaque formation. ${ }^{11}$

We hypothesized that the LITA would have lower VV density in normal animals and attenuated response when exposed to hypercholesterolemia in comparison with the coronary arteries. Hence in the current study micro-CT 

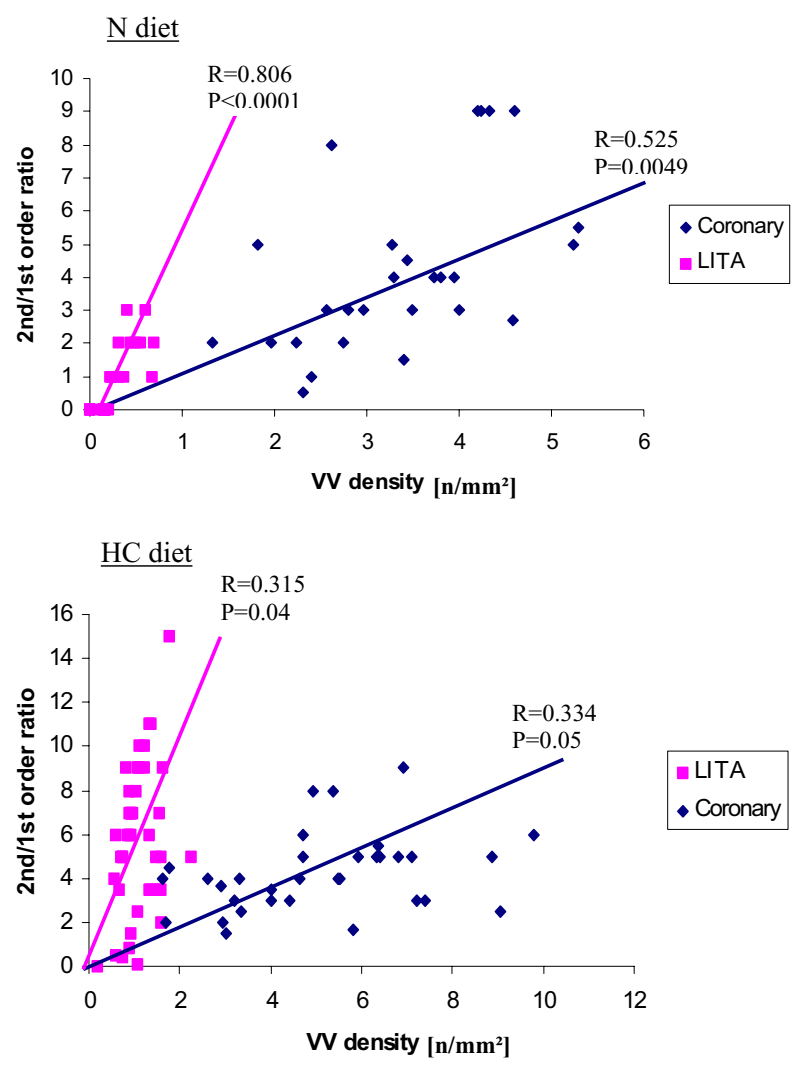

Figure 2. Correlation between second-order/first-order VV ratio and VV density in the coronary artery and the left internal thoracic artery (LITA) of pigs maintained on normal diet $(N)$ and a high-cholesterol diet (HC) for 12 weeks.

technology was applied to characterize the structure of the adventitial VV in the LITA in comparison with that seen in the coronary artery under normal and hypercholesterolemic conditions. Specifically, vessel lumen area, VV spatial density, distribution, diameter of the first and second order, and second-order/first-order VV ratio are compared.

\section{Methods}

\section{Animals}

The institutional animal care and use committee approved the study. Experiments were conducted on juvenile domestic crossbred pigs (weight, 55-65 kg; Larson Farms, Seargent, Minn) placed on a normal chow diet $(n=6)$ or on a hypercholesterolemic $\operatorname{diet}$ (HC diet; $\mathrm{n}=6 ; 2 \%$ cholesterol, $15 \%$ lard, TD 93296, Harlan Teklad, Madison, Wis) for 12 weeks. The swine is an established model to study human disease because its cardiovascular anatomy and physiology are comparable with those of human subjects. ${ }^{12}$ As we have previously demonstrated, feeding a high-cholesterol diet leads to a gradual increase in serum concentrations of total and low-density lipoprotein cholesterol over time, as well as accumulation of lipids in the intima and endothelial dysfunction before formation of atherosclerotic lesions. ${ }^{9}$

After the completion of the study period, the animals were killed with an overdose of pentobarbital sodium $(10 \mathrm{~mL}$ of Slee-

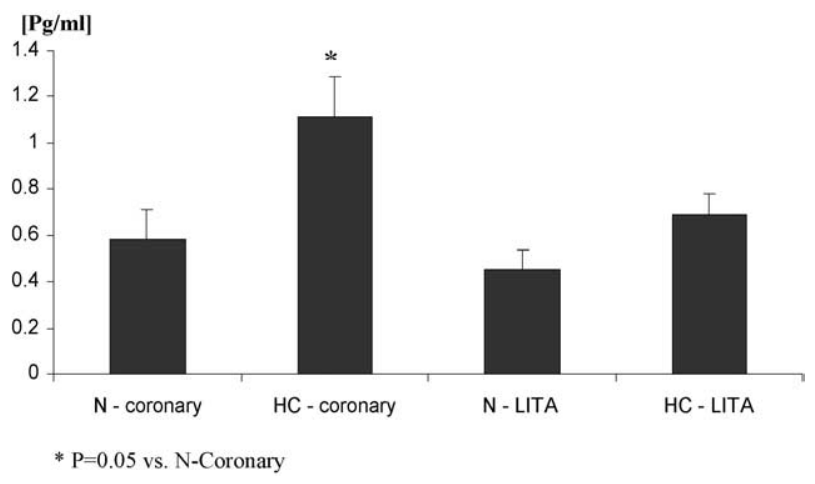

Figure 3. Enzyme-linked immunosorbent assay for VEGF in the coronary artery and left internal thoracic artery (LITA) of pigs maintained on a normal diet $(N)$ and a high-cholesterol diet (HC) for 12 weeks. An increase in VEGF expression in both vascular beds in response to the HC diet is observed.

paway, Fort Dodge Laboratories, Sligo, Ireland). Coronary arteries and LITAs were harvested immediately after death.

\section{Micro-CT Analysis}

As described previously, ${ }^{13}$ a glass cannula was advanced into the most proximal part of each artery, followed by injection of $500 \mathrm{~mL}$ of heparinized saline $(0.9 \%$ sodium chloride with $5000 \mathrm{IU}$ of heparin) at an infusion rate of $10 \mathrm{~mL} / \mathrm{min}$ to clear the circulation from remaining blood and microthrombi. Next, a low-viscosity (20 cP) lead chromate-doped silicone polymer (Microfil, MV-122; Canton Biomedical Products, Newington, Conn) was injected into the regional circulation at a rate of $0.6 \mathrm{~mL} / \mathrm{min}$ (pressure, $70 \mathrm{~mm}$ $\mathrm{Hg}$ ) until the polymer emerged from the veins. After overnight refrigeration at $4{ }^{\circ} \mathrm{C}$ to allow polymerization of the compound, the arteries were carefully dissected, preserving the adventitia intact, and placed in a $36.5 \%$ formaldehyde solution. Subsequently, specimens were placed in glycerin solution at increasing concentrations (30\%, 50\%, $75 \%$, and $100 \%$ changed at 24-hour intervals) for dehydration of the segments and thereafter rinsed with acetone, air-dried for 24 hours, and embedded in a paraffin mold for 3-dimesnional (3D) Micro-CT imaging.

The scan yielded an image with a 3D matrix of $42-\mu \mathrm{m}$ cubic voxels with 16 bits of gray scale (Figure 1). Data analysis was performed with Analyze software (Biomedical Imaging Resource, Rochester, Minn). On average, 6 to 12 cross-sections at $50-\mu \mathrm{m}$ intervals in areas between branch points were chosen for regionof-interest analysis. The area used for VV analysis was determined as previously described, normalized to the vessel lumen area, and designated the vessel wall area. ${ }^{14}$ The host vessel and the VV were manually traced and measured in this area on each cross-section, yielding the following parameters: vessel lumen area, vessel wall area, VV count, VV spatial density (ie, number of $\mathrm{VV} / \mathrm{mm}^{2}$ vessel wall area), mean diameter of first- and second-order $\mathrm{VV}$, and second-order/first-order VV ratio. First-order VV was defined as that originating from the vessel lumen and running longitudinally, and second-order VV was defined as that originating from firstorder VV and running circumferentially. ${ }^{15}$ 

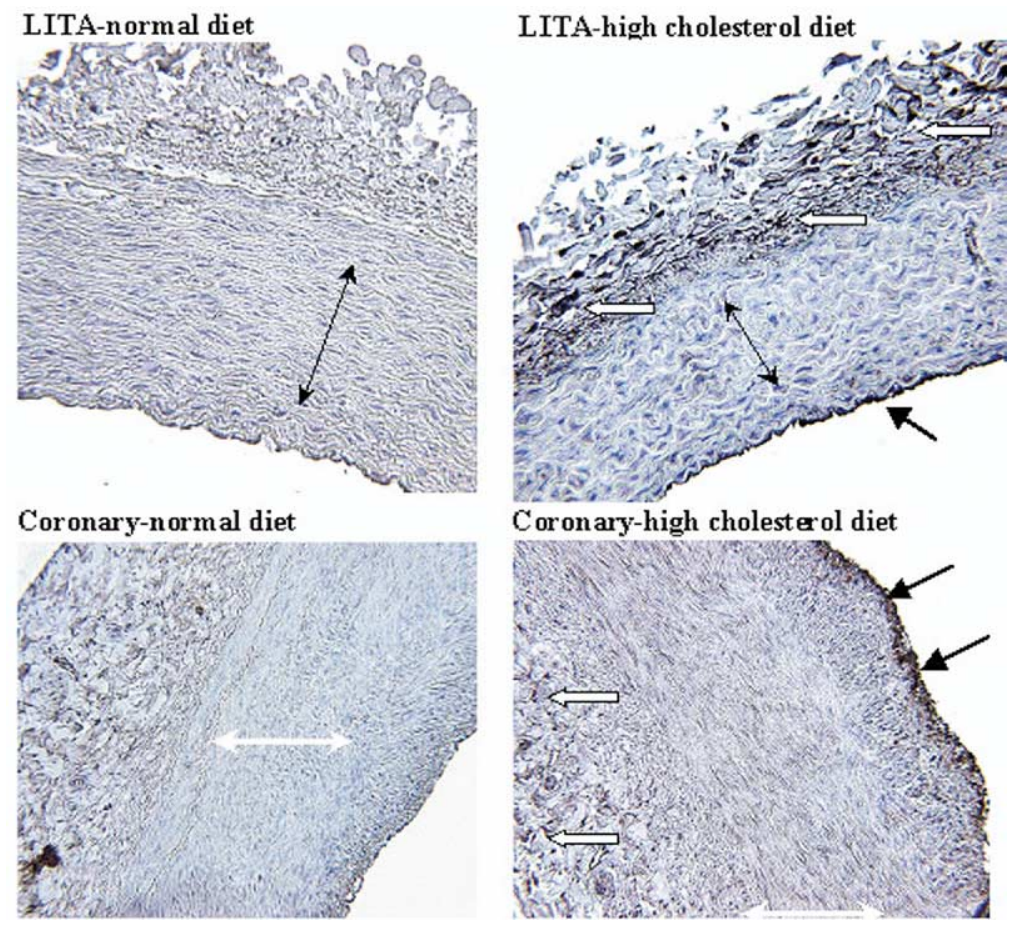

Figure 4. Immunohistochemistry for NF- $\kappa$ B (subunit p65). NF- $\kappa$ B is localized in the endothelial cells (black arrows) and in the adventitia (white arrows) of both vessels from pigs receiving a high-cholesterol diet. Vessels from pigs receiving a normal diet showed minimal staining for NF- $\kappa$ B. The left internal thoracic artery (LITA) contains a large number of elastic laminae in its media (black double-headed arrows) and few smooth muscle fibers, whereas the coronary artery contains a high amount of smooth muscle fibers (white double-headed arrows) and few elastic laminae.

\section{Staining for Nuclear Factor $\kappa B$ (Subunit p65)}

After embedding in paraffin, cross-sections of the arteries were mounted on slides. After deparaffinizing and hydrating, the tissue presence of nuclear factor $\kappa \mathrm{B}(\mathrm{NF}-\kappa \mathrm{B})$ was assessed as previously reported. ${ }^{16}$ Nonspecific protein-binding sites were blocked for 20 minutes at room temperature (protein block, DAKO, Carpinteria, Calif), followed by an overnight incubation at $4^{\circ} \mathrm{C}$ with the primary antibody polyclonal anti-p65 (1:25, Zymed Laboratories, Inc, San Francisco, Calif). The sections were subsequently covered with secondary antibodies conjugated to a horseradish peroxidaselabeled polymer for 30 minutes at room temperature (Envision) and then stained with diaminobenzidine (Vector Laboratories, Burlingame, Calif). Counterstaining was performed with hematoxylin before the sections were mounted in aqueous glycerol gelatin medium. In each group of slides, one slide served as a negative control by using rabbit $\mathrm{IgG}$.

Stained slides were viewed under a microscope (Olympus, Leeds Precision Instruments, North Minneapolis, Minn), and pictures were taken with an imaging program (SPOT Advanced 3.3, Diagnostic Instruments, Inc, Sterling Heights, Mich ).

\section{Enzyme-Linked Immunosorbent Assay for Vascular Endothelial Growth Factor}

Arterial segments, being snap-frozen immediately after removal, were homogenized with a tissue homogenizer and a lysis buffer, as previously described. ${ }^{17}$ Vascular endothelial growth factor (VEGF) was measured by means of enzyme-linked immunosorbent assay (Quantikine Human VEGF Immunoassay, R\&D Systems, Inc, Minneapolis, Minn) that detects soluble VEGF within an equal amount of total protein extracted from the tissue samples. Assays were conducted according to the manufacturer's directions, and standards and samples were tested in duplicate. Results were calculated from a standard curve, generated by an 8-parameter logistic curve fit, and expressed in picograms per milliliter.

\section{Statistical Analysis}

Continuous data are expressed as means \pm SE. Group comparison was based on $t$ tests for normal groups and Mann-Whitney rank sum tests for nonnormal groups. Pearson product moment correlation was used for correlation assessment.

\section{Results}

\section{Lipid Profile}

The HC group had a significantly higher level of total cholesterol $(379.3 \pm 33.2$ vs $72.6 \pm 4.8 \mathrm{mg} / \mathrm{dL}, P<.001)$, low-density lipoprotein $(272.2 \pm 28.9$ vs $35.4 \pm 4.6 \mathrm{mg} / \mathrm{dL}$, $P<.001)$, high-density lipoprotein $(97.9 \pm 8.4$ vs $32.5 \pm$ $3.4 \mathrm{mg} / \mathrm{dL}, P<.001)$, and triglycerides $(34.5 \pm 7.1$ vs 23.8 $\pm 2.4 \mathrm{mg} / \mathrm{dL}, P=.043)$ versus the normal group. 


\section{Dimensional Analysis of the Vessel Wall and VV}

Lumen area. The luminal area of the LITAs and coronary arteries was similar in the normal group. In the HC group the luminal area of the LITAs was significantly higher than the luminal area of the coronary arteries, as well as the luminal area of the LITAs in the normal group. No difference was observed between the luminal area of the coronary arteries in pigs receiving the $\mathrm{HC}$ diet and those receiving the normal diet (Table 1).

Vessel wall area, $V V$ count, and $V V$ density. As displayed in Table 1, in the normal group the LITAs had a significantly higher vessel wall area with a significantly lower VV count than the coronary arteries, resulting in a lower VV density. A significantly higher vessel wall area, VV count, and VV density was observed in both vascular beds in the HC group than in the normal group, being more pronounced in the LITAs. However, VV density remained significantly lower in the LITAs than in the coronary arteries (Table 1).

Second-order/first-order $V V$ ratio. In the normal group the second-order/first-order VV ratio was lower in the LITAs compared with that seen in the coronary arteries (Table 1). This relationship was inverted in the $\mathrm{HC}$ group, with a significantly higher second-order/first-order VV ratio in the LITAs. A significantly positive correlation was found between VV density and the second-order/first-order VV ratio in both arteries in both groups (Figure 2). The positive correlation likely reflects the fact that a higher VV density is due to an increase in the second-order VV count rather than first-order VV.

$\boldsymbol{V} \boldsymbol{V}$ diameter. First-order and second-order VV diameters were not different between the LITAs and the coronary arteries in the normal group. On the other hand, first-order and second-order VV diameter of the LITAs in the HC group were larger than that of the LITAs in the normal group, as well as that of the coronary arteries in the HC group (Table 1).

\section{Vascular Endothelial Growth Factor}

As displayed in Figure 3, VEGF levels were not significantly different between the coronary arteries and the LITAs in the animals receiving the normal diet, as well as in the animals receiving the HC diet. An increase in VEGF levels was observed in both vessels when exposed to hypercholesterolemia; however, this increase was significant only in the coronary arteries $(P=.05)$.

\section{Nuclear Factor $\kappa B$}

Immunohistochemistry localized NF- $\kappa \mathrm{B}$ (subunit p65) to the endothelial cells and the adventitia of the LITAs and the coronary arteries from pigs receiving an $\mathrm{HC}$ diet (Figure 4). Vessels from pigs receiving a normal diet showed minimal staining for NF- $\kappa$ B.
Atherosclerotic lesions were not observed in any of the vessels studied on histologic cross-section. Figure 4 further illustrates the significant structural difference between the 2 vascular beds. Coronary arteries are muscular, and their media contain a large number of smooth muscle fibers with few elastic laminae, whereas LITAs are elastic arteries with a large number of elastic laminae and few smooth muscle fibers in the media.

\section{Discussion}

The current study demonstrates a significant difference in the structure of the adventitial VV between coronary arteries and LITAs in animals receiving both normal and HC diets. Combined with the established differences in atherosclerosis prevalence between these 2 vascular beds, the results suggest a possible pathophysiologic role for the adventitial VV in atherogenesis.

Numerous in vivo and in vitro animal experiments have been done to study the biologic behavior of the internal thoracic arteries. ${ }^{18}$ However, despite recent studies,${ }^{6}$ there is a lack of information regarding the role of the adventitial $\mathrm{VV}$ in atherosclerosis. Micro-CT is a novel technology that enables us to outline the 3D anatomy of the VV. We have previously demonstrated that VV spatial density increases in hypercholesterolemic porcine coronary arteries, suggesting a significant role to the adventitial $\mathrm{VV}$ in the early atherosclerotic remodeling process before plaque formation. ${ }^{11}$

In the current study we applied the micro-CT technology to the coronary arteries and LITAs from pigs receiving normal and $\mathrm{HC}$ diets. In the normal group VV spatial density was significantly lower in the LITAs than in the coronary arteries. Exposure to an $\mathrm{HC}$ diet resulted in an increase in VV density in both vascular beds, and the HC group coronary VV density was significantly higher compared with LITA VV density. Second-order/first-order VV ratio in the LITA became significantly higher in the HC group. These findings, together with the positive direct correlation between VV spatial density and the secondorder/first-order VV ratio indicate that second-order VV proliferation accounts for most of the increased VV density. In line with the anoxemia theory of atherosclerosis, ${ }^{19}$ this anatomic difference might be the result of vessel wall exposure to different oxygen tensions. This is supported by the increase in VEGF levels in both vessels after an HC diet. $\mathrm{NF}-\kappa \mathrm{B}$ transcriptionally regulates other proangiogenic factors, as well as the inflammatory response. Immunohistochemistry revealed increased expression of NF- $\kappa \mathrm{B}$ in both vascular beds in response to the $\mathrm{HC}$ diet. This inflammatory marker is observed in the endothelial cells, as well as in the adventitia-VV area, supporting its role in the inflammation process during early atherosclerosis. 
There was an increase in the lumen area of the LITAs in the $\mathrm{HC}$ group that was not detected in the coronary arteries. These differences might imply that the remodeling process in the LITA is different and might be more effective than that in the coronary artery. The 2 vascular beds are histologicaly different. Coronary arteries are muscular arteries, and their media contain few elastic laminae and a large amount of smooth muscle fibers. The LITA, in contrast, has an elastic structure; its media contain few muscle fibers and a large number of thick elastic laminae. ${ }^{20}$ In the current study we observed an increased lumen area in response to the HC diet only in the LITA, which could be related to the elastic structure of the LITA.

Previous study in apolipoprotein E-deficient mice shows that antiangiogenic therapy not only reduced plaque neovascularization but eventually plaque growth. ${ }^{21}$ Furthermore, inhibition of angiogenesis has been shown to reduce macrophages in the plaque and around the $\mathrm{VV} .^{22}$ In the current study the absolute density of the VV was significantly lower in the LITA than in the coronary artery, hence exposing the LITA to inflammatory factors to a lesser degree. The significantly low VV spatial density observed in the LITAs in the normal group, as well as in the HC group, might underlie the low incidence of atherosclerosis in this vessel by rendering the vessel less susceptible to endothelial dysfunction, angiogenesis, and inflammation.

This suggested pathophysiologic mechanism is in line with the observation of a very low incidence of atherosclerosis in the thoracic vessel. ${ }^{4}$ Nevertheless, further investigation in human tissue exploring VV structure is warranted to further elucidate the physiologic characteristics of vessels with a low incidence of atherosclerosis.

In summary, the current study demonstrates heterogeneity in the structure of the VV between the coronary artery and LITA circulation. Moreover, in response to an HC diet, there was a differential response of VV proliferation. This study supports a role for the adventitial VV in the differential expression of atherosclerosis in different vascular beds.

\section{References}

1. American Heart Association. Heart disease and stroke statistics-2003 update. 2003;5-21. Dallas (TX): American Heart Association; 2002.

2. Pasterkamp G, Schoneveld AH, Hillen B, Banga JD, Haudenschild $\mathrm{CC}$, Borst C. Is plaque formation in the common carotid artery representative for plaque formation and luminal stenosis in other atherosclerotic peripheral arteries? A post mortem study. Atherosclerosis. 1998;137:205-10.

3. Vink A, Schoneveld AH, Poppen M, de Kleijn DP, Borst C, Pasterkamp G. Morphometric and immunohistochemical characterization of the intimal layer throughout the arterial system of elderly humans. J Anat. 2002;200:97-103.

4. Julke M, von Segesser L, Schneider J, Turina M, Heitz PU. Degree of arteriosclerosis of the internal mammary artery and of the coronary arteries in 45-to-75-year-old men. An autopsy study. Schweiz Med Wochenschr. 1989;119(36):1219-23.

5. Cameron A, Davis KB, Green G, schaff HV. Coronary bypass surgery with internal-thoracic-artery grafts: effects on survival over a 15-year period. N Engl J Med. 1996;334:216-9.

6. Wilcox JN, Scott NA. Potential role of the adventitia in arteritis and atherosclerosis. Int J Cardiol. 1996;54(suppl):S21-35.

7. Booth RFG, Martin JF, Honey AC, Hassall DG, Beesley JF, Moncada S. Rapid development of atherosclerotic lesions in the rabbit carotid artery induced by perivascular manipulation. Atherosclerosis. 1989; 76:257-68

8. Barger AC, Beeukwes R III, Lainey LL, Silverman KJ. Hypothesis: vasa vasorum and neovascularization of human coronary arteries. N Engl J Med 1984;310:175-7.

9. Lerman A, Ritman EL. Evaluation of microvascular anatomy by micro-CT. Herz. 1999;24:531-3.

10. Kwon HM, Sangiorgi G, Ritman EL, et al. Enhanced coronary vasa vasorum neovascularization in experimental hypercholesterolemia. J Clin Invest. 1998;101(8):1551-6.

11. Herrmann J, Lerman LO, Rodriguez-Porcel M, et al. Coronary vasa vasorum neovascularization precedes epicardial endothelial dysfunction in experimental hypercholesterolemia. Cardiovasc Res. 2001;51: 762-6.

12. Bloor CM, White FC, Roth DM. The pig as a model of myocardial ischemia and gradual coronary artery occlusion. In: Swindle MM, Moody DC, Phillips LD, editors. Swine as models in biomedical research. Ames (IA): Iowa State University Press; 1992. p. 163-75.

13. Wan SY, Ritman EL, Higgins WE. Multi-generational analysis and visualization of the vascular tree in $3 \mathrm{D}$ micro-CT images. Comput Biol Med. 2002;32:55-71.

14. Edelman ER, Nugent MA, Smith LT, Karnovsky MJ. Basic fibroblast growth factor enhances the coupling of intimal hyperplasia and proliferation of vasa vasorum in injured rat arteries. J Clin Invest. 1992; 89(2):465-73.

15. Zamir M, Silver MD. Vasculature in the walls of human coronary arteries. Arch Pathol Lab Med. 1985;109:659-62.

16. Wilson SH, Best PJM, Edwards WD, et al. Nuclear factor- $\kappa \mathrm{B}$ immunoreactivity is present in human coronary plaque and enhanced in patients with unstable angina pectoris. Atherosclerosis. 2002;160:147-53.

17. Rodriguez-Porcel M, Lerman LO, Holmes DR Jr, Richardson D, Napoli C, Lerman A. Chronic antioxidant supplementation attenuates nuclear factor- $\kappa \mathrm{B}$ activation and preserves endothelial function in hypercholesterolemic pigs. Cardiovasc Res. 2002;53:1010-8.

18. Kouchi Y, Onuki Y, Wu MH-D, Shi Q, Sauvage LR. Effects of altered blood flow on the caliber and morphology of the internal thoracic artery in the dog. J Thorac Cardiovasc Surg. 1997;113:114-20.

19. Gainer JL. Hypoxia and atherosclerosis: re-evaluation of an old hypothesis. Atherosclerosis. 1987;68:263-6.

20. Barry MM, Foulon P, Touati G, et al. Comparative histological and biometric study of the coronary, radial and left internal thoracic arteries. Surg Radiol Anat. 2003;25:284-9.

21. Moulton KS, Heller E, Konerding MA, Flynn E, Palinski W, Folkman J. Angiogenesis inhibitors endostatin or TNP-470 reduce intimal neovascularization and plaque growth in apolipoprotein E-deficient mice. Circulation. 1999;99:1726-32.

22. Moulton KS, Vakili K, Zurakowski D, et al. Inhibition of plaque neovascularization reduces macrophage accumulation and progression of advanced atherosclerosis. Proc Natl Acad Sci U S A. 2003;100: 4736-41. 